\title{
Conducta prosocial en niños de padres separados en relación con estilos de afrontamiento
}

\author{
Prosocial behavior in children of separated \\ parents regarding coping styles
}

\author{
Jesús Percy Romero Croce ${ }^{1}$, Liz Katerine Flower Quenta*, \\ Roxana Shirley Huamanttica Valverde ${ }^{* *}$ \\ Escuela Profesional de Psicología, \\ Universidad de San Martín de Porres, Perú
}

\section{Resumen}

El propósito del presente artículo es establecer cómo los estilos de afrontamiento en niños influyen en su comportamiento prosocial, particularmente en aquellos afectados por el cambio estructural de la familia derivado del divorcio o separación de sus padres. En la actualidad, las estadísticas evidencian el aumento de los divorcios como secuela de la pandemia. El divorcio altera la parte psicoafectiva de los hijos, quienes manifiestan dificultades a nivel académico, cambios de estado de ánimo y de comportamiento, junto a problemas de interrelación con los demás. Asimismo, como cada familia funciona con una dinámica propia, también serán diferentes los modos de afrontar y recuperar la salud emocional, psicológica y social. Apreciaremos la relación de la conducta prosocial y los estilos de afrontamiento en niños de padres separados, teniendo en cuenta que esta se relaciona con la empatía, y consideraremos la influencia positiva o negativa de los miembros de la familia.

1 Q.E.P.D. Docente, catedrático, comprometido con el desarrollo y difusión de investigaciones científicas y el quehacer profesional. Nuestro mayor reconocimiento por sus enseñanzas y consejos en vida compartidos con todos sus alumnos, colegas y amigos. Una amistad que perdurará en el tiempo con recuerdos memorables en todos sus seres queridos.

Este es un artículo Open Access bajo la licencia Creative Commons Atribución-NoComercial-Compartirlgual 4.0

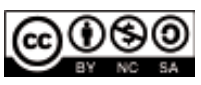


Palabras clave: familia, dinámica familiar, conducta prosocial, estilos de afrontamiento.

\begin{abstract}
The purpose of this article is to establish how children's coping styles influence their prosocial behavior, particularly in those affected by the structural change in the family derived from divorce or separation from their parents. Currently, statistics show the increase in divorces as a consequence of the pandemic. Divorce alters the psycho-affective part of the children, who manifest difficulties at the academic level, changes in mood and behavior, along with problems of interrelation with others. Likewise, as each family functions with its own dynamics, the ways of facing and recovering emotional, psychological, and social health will also be differents. We will appreciate the relationship of prosocial behavior and coping styles in children of separated parents, taking into account that this is related to empathy, and we will consider the positive or negative influence of family members.
\end{abstract}

Keywords: family, family dynamics, prosocial behavior, coping styles.

\title{
Introducción
}

González (2000) afirma que la conducta prosocial puede ser entendida como todo comportamiento social positivo, al tiempo que propone la hipótesis de empatía-altruismo. Los autores en mención desarrollan esta propuesta considerando que la preocupación empática constituye un intento de reducir el sufrimiento ajeno y que la conducta altruista es toda acción espontánea realizada de manera desinteresada hacia los demás.

En este contexto, Carlo et al. (2010) señalan que el comportamiento prosocial es un factor moderador de la agresividad y constituye una disposición que favorece la adaptación y el desarrollo de las habilidades sociales, en tanto que Molero et al. (1999) advierten que el malestar personal de los niños está negativamente relacionado con la conducta prosocial. 
En una investigación comparativa de respuestas a la privación parental entre niños de padres separados y huérfanos con respecto a niños de familias intactas se hallan diferencias marcadas. En los dos primeros grupos se aprecia una percepción de clima sociofamiliar más empobrecido, afectando la personalidad, el estado de humor y la adaptación, generando sentimientos de depresión e insatisfacción familiar (Bengoechea, 1996).

Los estilos parentales ejercen una influencia directa en la percepción de empatía con respecto a los padres y en el establecimiento de la conducta prosocial en los niños.

De este modo, según Richaud et al. (2011), los estilos parentales de aceptación inciden en la conducta prosocial, mientras que el control patológico tiene una influencia negativa. La negligencia parental pareciera ejercer una influencia directa, en tanto que la indiferencia parental no favorece la percepción de empatía (el niño asume que si no le importa a nadie, pues tampoco a él le interesan los demás).

Las estrategias de afrontamiento hacen referencia al proceso cognitivo y conductual único de cada persona, que se expresa en su forma de atender a las diversas demandas generadas en su entorno (Lazarus y Folkman, 1986).

\section{Marco teórico}

Los estilos parentales son pautas de crianza de los hijos asumidos por sus padres, estilos de relación relativamente estables. Según Davis, 2016; Merino y Arndt, 2004; Oudhof et al. 2012: y Richaud et al. 2011, estos pueden ser clasificados del modo siguiente:

- Estilo parental autoritativo o democrático. Caracterizado por el apoyo emocional, con límites establecidos, autonomía y respeto mutuo, estimulando la toma de decisiones e independencia, guiados por los padres.

- Estilo parental autoritario. Forma de educar exigente de los padres con mucho control e inflexibilidad en el actuar, generando en los hijos dependencia e inseguridad. 
- Estilo parental permisivo. Carencia de normas y límites hacia los hijos, no existen responsabilidades ni demandas por parte de los padres, quienes son indulgentes y evitan situaciones de conflicto.

- Estilo parental negligente. Padres sin compromiso afectivo, psicológico y falta de interés por atender las necesidades de sus hijos.

Los estilos parentales descritos ejercen una influencia directa en las estrategias de afrontamiento asumidas por los niños. Así, las familias democráticas proponen un afrontamiento adaptado; las familias rechazantes, por el contrario, condicionan un afrontamiento desadaptado, generador de soledad frente a los pares, además de depresión; mientras que las familias autoritarias generan comportamientos de búsqueda de apoyo en personas ajenas a la familia, así como inseguridad (Richaud, 2005).

En este sentido, cuando los niños adoptan estilos de aproximación al problema, estos se asocian a resultados favorables de adaptación emocional; por el contrario, cuando se instalan estrategias de afrontamiento evitativo, este se asocia con síntomas depresivos, mayores niveles de comportamiento agresivo y ansiedad (Hernández y Gutiérrez, 2012; Morales-Rodríguez et al., 2012).

Ahora bien, si es evidente que la constelación familiar tiene una influencia directa en el desarrollo cognitivo y emocional de los niños, ¿̇uál es el impacto de la ruptura del vínculo parental, por divorcio o separación de los padres?

Para Cifuentes y Milicic (2012), la pérdida de la familia nuclear afecta el desempeño conductual y socioemocional, lo que se refleja en el ámbito social y escolar.

Muñoz-Ortega et al. (2008) y Bengoechea (1996) establecen que tal circunstancia genera una percepción de clima sociofamiliar empobrecido, pensamientos de preocupación por el futuro, inseguridad, temores y ansiedad, junto a sentimientos de depresión e insatisfacción familiar, rabia, tristeza y resentimiento.

Por su parte, Mora y Navarro (2012) definen a la familia como la base fundamental de la sociedad que influye en el desarrollo de los aspectos 
cognitivo, afectivo, educativo y socioeconómico de los hijos. Por tanto, es imprescindible conocer la influencia de las relaciones familiares en la primera etapa de vida, considerando las características propias y particulares de la dinámica familiar a través de su interrelación e intercomunicación (Marín et al., 2019).

En la investigación de Orgilés y Samper (2011) se trata el cambio estructural dentro de la familia producido por el divorcio y que origina una desorganización a nivel psicológico, repercutiendo de manera negativa en los niños, quienes presentan miedos intensos en el colegio, ansiedad por la ausencia de uno de los padres, así como cambios en su comportamiento habitual.

El estudio realizado por Granda y Solano (2016) expone los diferentes aspectos negativos que trae consigo la separación de los progenitores en la dinámica familiar. Tener que adaptarse a otra estructura moviliza en el niño alteraciones de ánimo, como tristeza y ansiedad. De la misma forma, resistirse a los cambios, ya que la familia no está consolidada, se aprecia en la conducta y el rendimiento inadecuados del niño.

En una investigación internacional donde se analizó la percepción y valoración de los niños sobre la separación o divorcio de los padres en diferentes contextos, tanto rurales como urbanos, se llegó a la siguiente conclusión: los primeros tienen una apreciación más positiva en torno al tema de la separación o divorcio, refiriendo que si los padres se separan no es por el hecho de no amar a sus hijos; mientras que los niños de contextos urbanos perciben de forma negativa la ruptura familiar (Pérez y Luján, 2015).

En nuestro país, el Instituto Nacional de Estadística e Informática (INEI, 2020b) realizó una investigación en conjunto con el Registro Nacional de Identificación y Estado Civil (RENIEC), informando que en el año 2018 se inscribieron 92440 matrimonios civiles a nivel nacional, siendo Lima el departamento con el mayor porcentaje: $43.4 \%$ (40 141), mientras que Madre de Dios (3\%), la Provincia Constitucional del Callao y Tumbes (4\% cada uno) fueron los que presentaron menor proporción. 
Un panorama diferente se observó en el reporte posterior presentado solo por el INEI (2020a), con 16485 divorcios a nivel nacional, siendo Lima el departamento con el mayor porcentaje (62.3\%).

\section{Figura 1}

Divorcios inscritos por año, 2015-2019 (INEI, 2020a)

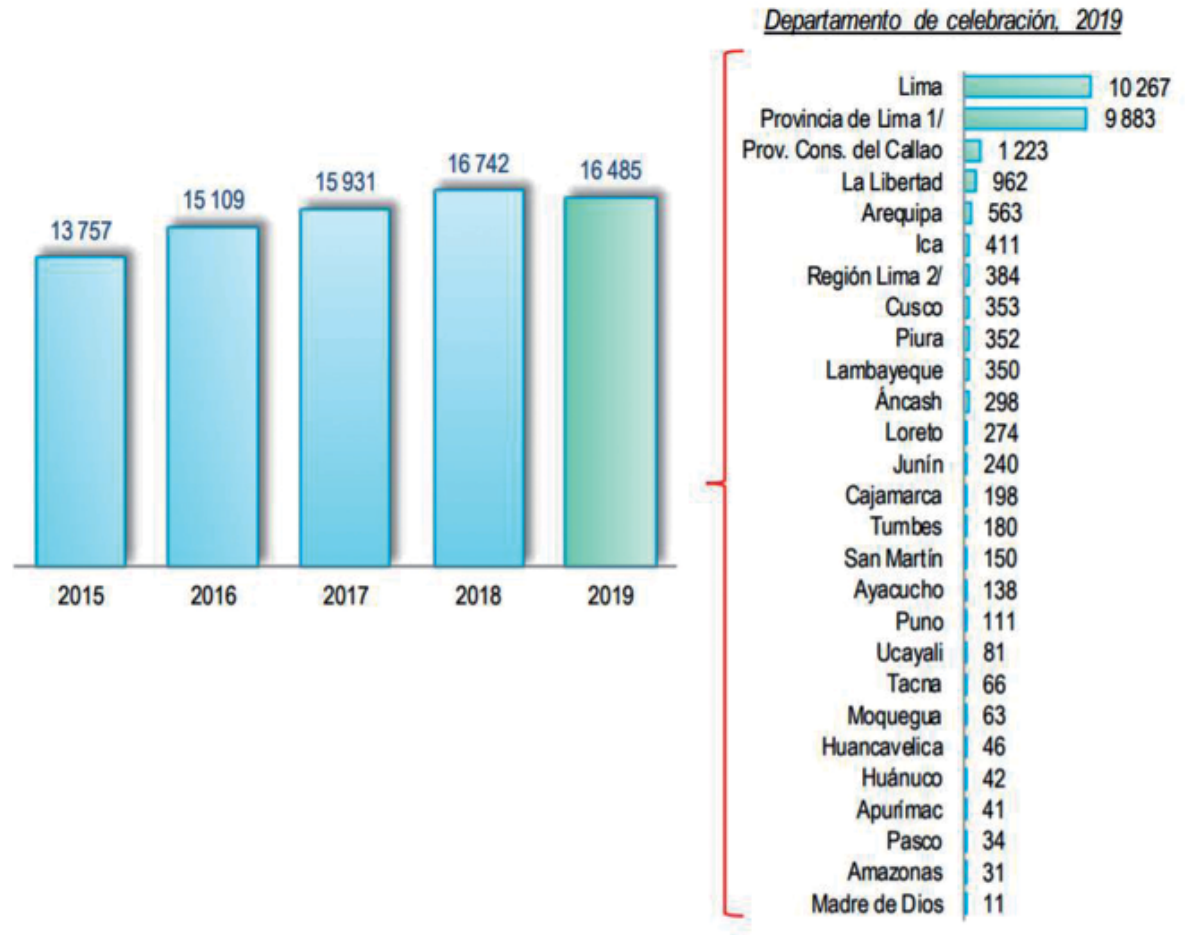

El incremento de la tasa de separación o divorcio de los padres repercute en el bienestar de los hijos y en la estructura familiar debido a los constantes conflictos entre los cónyuges y el incumplimiento de las pautas o acuerdos de crianza, ocasionando deterioro en la salud física, emocional y psicológica de los niños a corto, mediano y largo plazo (Orgilés y Samper, 2011).

Por ello, es importante realizar estudios que identifiquen el grado de afectación en la calidad de vida de los hijos ante el divorcio o separación 
de los padres y su relación con el desarrollo de conductas prosociales y estilos de afrontamiento (Núñez et al., 2017). La capacidad de los niños para afrontar esta situación compleja e inestable reconstruyendo su esquema mental redunda en la adopción de mejores estrategias de afrontamiento (Radovanovic, 1993); en su defecto, experimentan sentimientos negativos y problemas de conducta (Lengua y Sandler, 1996).

\section{Conclusiones}

El divorcio es un fenómeno que se va acrecentando a nivel mundial y nuestro país no es la excepción, más aún durante la pandemia, y esto se debe a los cambios emocionales, psicosociales, económicos y culturales, entre otros, que se establecen en la dinámica familiar entre los padres y los hijos. La ausencia de alguno de los padres, sumada a la inadecuada manera de resolver los conflictos, influye en su comunicación e interacción y en su manera de afrontar la situación de divorcio.

La conducta prosocial está íntimamente relacionada con la empatía y las estrategias de afrontamiento que son parte de nuestra vida diaria para resolver problemas y toma de decisiones, pensando no solo a nivel individual sino en el colectivo del bien común, siendo la familia la base fundamental para mejorar la calidad de vida (emocional-psicológica) entre todos.

Si bien encontramos en algunas investigaciones aspectos negativos como el bajo rendimiento escolar, cambios de estado de ánimo y comportamiento inadecuado en niños con padres divorciados, existen otras enfocadas en áreas positivas, que pretenden desarrollar y mejorar la interacción entre los miembros de la familia y ser capaces de afrontar la separación.

La psicología brinda herramientas que facilitan este afrontamiento y el desarrollo de conductas prosociales mediante la resolución de problemas y el acompañamiento profesional durante el proceso de la separación y/o divorcio, con el fin de disminuir el grado de afectación entre los integrantes de la familia, siendo los hijos quienes presencian en mayor medida los conflictos de los padres. 


\section{Referencias}

Bengoechea, P. (1996). Un análisis comparativo de respuestas a la privación parental en niños de padres separados y niños huérfanos en régimen de internado. Psichotema, 8(3), 597-608. http://hdl.handle.net/ 10651/29285

Carlo, G., Mestre, M. V., Samper, P., Tur, A. y Armenta, B. E. (2010). Feelings or cognitions? Moral cognitions an emotions as longitudinal predictors of prosocial an agressive behaviour. International Journal of Behavioural Development, 34, 1-9. https://doi.org/10.1016/j.paid.2010.02.010

Cifuentes, O. y Milicic, N. (2012). Crisis en la infancia: ¿qué piensan, sienten y dicen los niños sobre la separación de sus padres? Universitas Psychologica, 11(2), 469-480. https://doi.org/10.11144/Javeriana.upsy11-2.cips

Davis, M. (2016). Parenting styles: The ultimate tips on parenting styles for raising children in the 21st century by applying parenting with purpose driven styles! CreateSpace Independent Publishing Plataform.

González, M. D. (2000). Conducta prosocial: evaluación e intervención. Morata.

Granda, M. K. y Solano, M. L. (2016). Divorcio de los padres y el cambio del comportamiento en los niños [Tesis de licenciatura, Universidad Técnica de Machala]. http://repositorio.utmachala.edu.ec/handle/48000/8315

Hernández, S. y Gutiérrez, L. (2012). Estilos de afrontamiento ante eventos estresantes en la infancia. Acta de Investigación Psicológica, 2(2), 687-698. https://www.redalyc.org/pdf/3589/358933341007.pdf

Instituto Nacional de Estadística e Informática (INEI). (2020a). Perú: Natalidad, mortalidad y nupcialidad 2019 (departamentos, provincia y distrito). https://www.inei.gob.pe/media/MenuRecursivo/publicaciones_digitales/Est/ Lib1766/libro.pdf

Instituto Nacional de Estadística e Informática (INEI). (2020b). En el país se celebraron más de noventa y dos mil matrimonios durante el año 2018. https://www.inei.gob.pe/prensa/noticias/en-el-pais-se-celebraron-mas-denoventa-y-dos-mil-matrimonios-durante-el-ano-2018-12057/

Lazarus, R. y Folkman, S. (1986). Estrés y procesos cognitivos. Martínez-Roca.

Lengua, L. J. y Sandler, I. N. (1996). Self-regulation as a moderator of the relation between coping and symptomatology in children of divorce. Journal of Abnormal Child Psychology, 24, 681-701. https://doi.org/10.1007/BF01664734

Marín, M., Quintero, P. A. y Rivera, S. C. (2019). Influencia de las relaciones familiares en la primera infancia. Poiesis (36), 164-183. https://doi.org/10.21501/16920945.3196

Merino, C. y Arndt, S. (2004). Análisis confirmatorio de la Escala de Estilos de Crianza de Steinberg: validez preliminar de constructo. Revista de Psicología de la PUC, 22(2), 187-214. https://doi.org/10.18800/ psico.200402.002

Molero, C., Candela, C. y Cortés, M. T. (1999). La conducta prosocial: una visión de conjunto. Revista Latinoamericana de Psicología, 31(2), 325-353. https://www.redalyc.org/pdf/805/80531207.pdf

Mora, W. A. y Navarro, D. E. (2012). Manifestaciones características de los sentimientos en adolescentes de 12 a 17 años al interior de sus familias del barrio Casa Blanca Norte [Tesis de especialización, Universidad de la Sabana]. http://hdl.handle.net/10818/2163

Morales-Rodríguez, F. M., Trianes, M. V., Blanca, M. J., Miranda, J., Escobar, M. y Fernández-Baena, F. J. (2012). Escala de Afrontamiento para Niños (EAN): propiedades psicométricas. Anales de Psicología, 28(2), 475-483. https://www.redalyc.org/articulo.oa?id=16723135018

Muñoz-Ortega, M. L., Gómez-Ayala, P. A. y Santamaría-Oglastri, C. M. (2008). Pensamientos y sentimientos reportados por los niños ante la separación de sus padres. Universitas Psychologica, 7(2), 347-356. http:// www.scielo.org.co/pdf/rups/v7n2/v7n2a04.pdf 
Núñez, C. S., Pérez, C. y Castro, M. (2017). Consecuencias del divorcio-separación en niños de edad escolar y actitudes asumidas por los padres. Revista Cubana de Medicina General Integral. 33(3), 296-309. http:/l scielo.sld.cu/scielo.php?script=sci_arttext\&pid=S0864-21252017000300003

Orgilés, M. y Samper, M. (2011). El impacto del divorcio en la calidad de vida de los niños de 8 a 12 años de edad en la provincia de Alicante. Gaceta Sanitaria, 25(6), 490-494. https://doi.org/10.1016/j.gaceta.2011.05.013

Oudhof, H., Rodríguez, B. y Robles, E. (2012). La percepción de la crianza en padres, madres e hijos adolescentes pertenecientes al mismo núcleo familiar. Liberabit, 18(1). 75-81. https://www.redalyc.org/pdf/686/68623931010.pdf

Pérez, D. y Luján, I. (2015). Percepción de niños de contextos rurales y urbanos sobre separación y divorcio. International Journal of Developmental and Educational Psychology, 1(1), 285-295. https://doi.org/10.17060/ ijodaep.2015.n1.v1.114

Radovanovic, H. (1993). Parental conflict and children's coping styles in litigating separated families: relationships with children's adjustment. Journal of Abnormal Child Psychology, 21(6), 697-713. https://doi.org/10.1007/ BF00916451

Richaud, M. C. (2005). Estilos parentales y estrategias de afrontamiento en niños. Revista Latinoamericana de Psicología, 37(1), 47-58. https://www.redalyc.org/pdf/805/80537102.pdf

Richaud, M. C., Lemos, V. y Mesurado, B. (2011). Relaciones entre la percepción que tienen los niños de los estilos de relación y de la empatía de los padres y la conducta prosocial en la niñez media y tardía. Avances en Psicología Latinoamericana, 29(2), 330-343. https://www.redalyc.org/pdf/799/79922588012.pdf 